\title{
Overexpression of HNF4a enhances umbilical cord mesenchymal stem cell function to treat acute liver failure in mice through macrophage polarization
}

\section{Yeping Yu}

Department of Liver Surgery, Renji Hospital, School of Medicine, Shanghai Jiao Tong University, Shanghai, China

\section{Qiqi Zhang}

Department of Liver Surgery, Renji Hospital, School of Medicine, Shanghai Jiao Tong University, Shanghai, China

\section{Ning Wu}

Department of Liver Surgery, Renji Hospital, School of Medicine, Shanghai Jiao Tong University, Shanghai, China

\section{Lei Xia}

Department of Liver Surgery, Renji Hospital, School of Medicine, Shanghai Jiao Tong University, Shanghai, China

\section{Jie Cao}

Department of Liver Surgery, Renji Hospital, School of Medicine, Shanghai Jiao Tong University, Shanghai, China

\section{Qiang Xia}

Department of Liver Surgery, Renji Hospital, School of Medicine, Shanghai Jiao Tong University, Shanghai, China

\section{Jie Zhao}

Department of Liver Surgery, Renji Hospital, School of Medicine, Shanghai Jiao Tong University, Shanghai, China

\section{Jianjun Zhang}

Department of Liver Surgery, Renji Hospital, School of Medicine, Shanghai Jiao Tong University, Shanghai, China

Hualian Hang ( $\sim$ hanghualian@shsmu.edu.cn)

Renji Hospital, School of Medicine, Shanghai Jiao Tong University, Shanghai, China

\section{Research}

Keywords: Acute liver failure, macrophages, mesenchymal stem cells, hepatocyte nuclear factor $4 a$ 
Posted Date: November 5th, 2020

DOI: https://doi.org/10.21203/rs.3.rs-26740/v2

License: (c) (i) This work is licensed under a Creative Commons Attribution 4.0 International License. Read Full License 


\section{Abstract}

Background and aim: Advances in research on stem cell therapy provide new feasible solutions for acute liver failure (ALF) treatment. Recent studies have demonstrated that the expression of hepatocyte nuclear factor $4 a(\mathrm{HNF} 4 \mathrm{a})$ reset within the damaged hepatocytes can restore normal physiological function. This study aimed to determine the role of human umbilical cord mesenchymal stem cells (HuMSCs) overexpressing HNF4a in ALF treatment.

Method: HuMSCs in vitro were isolated, cultured, and reprogrammed by the lentiviral expression of HNF4a (hereinafter referred to as HuMSC-HNF4a). HuMSC-HNF4a was intraperitoneally administered into the mice immediately after exposed to D-galactosamine/lipopolysaccharide (D-galN/LPS). The liver histological and serum biochemical analyses were performed to investigate the effects of HuMSC-HNF4a in ALF. Macrophages differentiation and cytokines secretion by HuMSCs were evaluated to elucidate the underlying mechanisms.

Results: HuMSC-HNF4a had more obvious therapeutic effects on ALF compared with negative control virus-transfected the HuMSCs (HuMSC-CON). In vitro, HuMSC-HNF4a promoted the polarization of liver macrophages (Kupffer cells) to the M2 phenotype, inhibited the inflammatory response of macrophages, and reduced the levels of inflammatory factors such as TNF- $a$ and IL-1 $\beta$ to reduce liver damage.

Conclusions: This study confirmed that the therapeutic effect of HuMSC-HNF4a on ALF was not the same as the previous passive support but as active intervention on excessive inflammation in the body. The findings provided new ideas for research and clinical practice in the future.

\section{Introduction}

Acute liver failure (ALF) is a clinical syndrome characterized by acute and severe liver damage, caused by various factors such as viral infection, toxic drugs, metabolic diseases, and alcohol-induced damage [1]. Orthotopic liver transplantation (OLT) is one of the most effective ways to treat ALF. However, this therapy is limited by the rarity of donor livers and the poor systemic condition of patients before surgery due to long waiting time [2]. Preliminary studies indicated that stem cells played a key role in treating ALF by trans-differentiating into hepatocytes to perform alternative functions, including promoting liver regeneration, reducing hepatocytes apoptosis, inhibiting liver fibrosis, and regulating the immune and inflammatory response [3-6]. Based on importance of immune responses in ALF, the immune regulation of stem cells may be the most important factor affecting the treatment of ALF [7].

Stem cells, especially mesenchymal stem cells (MSCs), are excellent genetic carriers due to their accessibility, strong differentiation potential, and proliferative capacity. Previous studies have confirmed that, the overexpression of thioredoxin-1 (Trx-1), insulin like growth factor 1 (IGF-1), hepatocyte growth factor (HGF) and other genes enhanced the protective effect of MSCs on ALF through reverse transcription and other technologies [8-10]. MSCs with the high expression of CXC chemokine receptor 
type 4 (CXCR4) could improve the quality of life and prolong the survival time of patients with ALF caused by small-for-size syndrome after liver transplantation.

Hepatocyte nuclear factor $4 a(\mathrm{HNF} 4 \mathrm{a})$ is a nuclear transcription factor previously shown to be lowly expressed in liver diseases such as liver tumors and ALF. Also, the physiological functions of hepatocytes can be restored by resetting the normal expression of HNF4a [11, 12]. Further, the overexpression of HNF4a in MSCs though gene transfection technology promoted the differentiation of MSCs into hepatocytes [13-15]. HNF4a is closely related to ALF and has strong regulatory effects on functions of MSCs, suggesting that MSCs with the high expression of HNF4a are expected to prossess potential curative effects on ALF.

Macrophages in the liver (Kupffer cells) can differentiate into M1 or M2 macrophages in response to various local microenvironments [16]. M1 macrophages mediate inflammatory damage and promote inflammation. Conversely, the M2 phenotype, which is characterized by anti-inflammatory factors, such as IL-4 and interleukin-10 (IL-10), and protective proteins (TGF- $\beta$ and VEGF), inhibits inflammation and repair tissue damage $[17,18]$. MSCs have been shown to promote M2 macrophage polarization and thus exert an anti-inflammatory effect by secreting IDO, PGE2, and so forth in response to environmental signals including elevated levels of IL-6 [19-21].

In this study, human umbilical cord-derived MSCs (HuMSCs) with overexpression of HNF4a (HuMSCHNF4a) were first established. Then, their therapeutic effects on lipopolysaccharide/D-galactosamine (LPS/D-GaIN)-induced ALF were observed to explore their therapeutic potential for ALF.

\section{Materials And Methods}

\subsection{Isolation and identification of HuMSCs}

This study was approved by the Institutional Review Board and Human Ethics Committee of Ren $\mathrm{Ji}$ Hospital, School of Medicine, Shanghai Jiao Tong University, Shanghai, China. All umbilical cords used in this study were obtained from healthy full-term cesarean section fetuse at Renji Hospital affiliated to Shanghai Jiao Tong University School of Medicine. Written consent for the use of samples for research purposes was obtained from all patients. HuMSCs were treated as previously described [14]. HuMSCs differentiated into chondrogenic (Cyagen Biosciences, Guangzhou, China), osteogenic (Cyagen Biosciences, Guangzhou, China), and adipogenic lineages (Cyagen Biosciences, Guangzhou, China), as indicated by positive type II collagen (Abcam, Cambridge, MA, USA, Cat. \#ab34712), Alizarin red, and Oil Red $O$ staining, respectively, after induced with a conditioned medium for 2-3 weeks. The expressioin patterns of CD73, CD90, CD105, CD34, CD45, major histocompatibility complex, class II, DR (HLA-DR) were detected by flow cytometry to evaluate the phenotypes of HuMSCs.

\subsection{Establishment and identification of HuMSC-HNF4a}


HuMSCs with stable overexpression of HNF4a, called HuMSC-HNF4a, were constructed as previously described [14]. HuMSCs transfected with a lentiviral vector containing only green fluorescent protein (HuMSC-CON) were treated as controls. The lentiviral transduction efficiency was detected by Western bot and Real-time quantitative reverse transcription-PCR.

\subsection{Flow cytometry}

Flow cytometry (BD Sciences) was performed to characterize the phenotypes of HuMSCs and macrophages. Antibodies against the human antigens CD11b (Cat. \#562721), CD68 (Cat. \#565594), CD80 (Cat. \#561134), CD206 (Cat. \#550889), CD73 (Cat. \#562430), CD90 (Cat. \#563070), CD105 (Cat. \#563803), CD34 (Cat. \#562383), CD45 (Cat. \#563792), and HLA-DR (Cat. \#562804) were purchased from BD Sciences, San Diego, CA, USA. Further, antibodies against the mouse antigens CD86 (BD Sciences, San Diego, CA, USA, Cat. \#553690) and CD206 (BD Sciences, San Diego, CA, USA, Cat. \#565250) were used to observe the effect of in vivo on macrophages phenotype. The data were analyzed using the BD CellQuest Pro software (V1.0.2).

\subsection{Animal}

Wild-type mice (C57BL/6J) were purchased from Shanghai Slark Experimental Animal Co., Ltd., and managed by the Animal Experimental Center of Renji Hospital, Shanghai Jiaotong University (Specific Pathogen Free grade). The experimental procedure was approved by the Animal Ethics Committee of Renji Hospital, Shanghai Jiao Tong University School of Medicine. Further, $2 \times 10^{6}$ HuMSC-HNF4a and HuMSC-CON were added to phosphate-buffered saline (PBS) solution. Mice aged 6 weeks were intraperitoneally injected with HuMSC-HNF4a cells, HuMSC-CON, or PBS $(10 \mathrm{~mL} / \mathrm{kg})$. D-GalN (700 mg/kg, Sigma-Aldrich, St. Louis, MO, Cat. \#G0500) and LPS (10 $\mu \mathrm{g} / \mathrm{kg}$, Sigma-Aldrich, St. Louis, MO, Cat. \#L2630) were administered via the abdominal cavity after $24 \mathrm{~h}$ of pretreatment. The survival status of mice was observed and recorded $4 \mathrm{~h}$ after LPS/D-GalN injection for $48 \mathrm{~h}$. It was recorded every half hour from $4 \mathrm{~h}$ to $12 \mathrm{~h}$ and every 6 hours between $12 \mathrm{~h}$ and $48 \mathrm{~h}$. The mice were anesthetized with $1 \%$ sodium 3 $\mathrm{h}$ after intraperitoneal injection of LPS/D-GalN. Blood and livers were collected subsequently. The blood samples were centrifuged at $5000 \mathrm{rpm}$ for $15 \mathrm{~min}$ to collect plasma. The plasma and liver tissues were frozen immediately in liquid nitrogen and stored at $-80^{\circ} \mathrm{C}$ until analysis.

\subsection{Real-time quantitative reverse transcription-PCR}

Total RNA was isolated from cultured HuMSCs using TRIzol reagent (Invitrogen Inc, CA, USA) and reversetranscribed into cDNA using a reverse transcription kit (Takara Bio USA, CA, USA). The PCR reaction was carried out in $20 \mu \mathrm{L}$ of a final volume containing $0.1 \mu \mathrm{M}$ of each forward and reverse primer, cDNA and 10 $\mu \mathrm{L}$ of SYBR Green PCR Master Mix (Takara Bio USA, CA, USA). DNA was amplified and analyzed using a StepOnePlus real-time PCR machine (Thermo-Fisher Scientific, Inc., Rochford, IL, USA).

\subsection{Western blot analysis}


To determine the expression of HNF4a, total protein was lysed with radio immunoprecipitation assay (RIPA) peptide lysis buffer (Beyotime Biotechnology, Jiangsu, China, Cat. \#P0013B) containing 1\% protease inhibitors (Thermo Fisher Scientific, Waltham, USA, Cat. \# A32953). Western blot analysis were performed according to anti- $\beta$-actin (Santa Cruz, CA, USA, Cat. \#sc-8432) and anti-HNF4a (Santa Cruz, CA, USA, Cat. \#sc-374229).

\subsection{Detection of serological indicators}

The enzyme activities of aspartate aminotransferase (AST) and alanine transaminase (ALT) in plasma were ditected with assay kits (Nanjing Jiancheng Bioengineering Institute, China). Enzyme-linked immunosorbent assay (ELISA) kits (R\&D Systems, Minneapolis, MN) were used to evaluate TNF-a and IL$1 \beta$ levels in plasma. HuMSC-CON and HuMSC-HNF4a were seeded in six-well plates at a density of $5 \times$ 105 well. When the cells reached $70 \%$ confluence, the remaining supernatant was washed with PBS. Fetal bovine serum (FBS)-free F12 (Gibco, MA, USA) was used to continue culture routinely. After $48 \mathrm{~h}$, the supernatant was collected into $15 \mathrm{~mL}$ centrifuge tubes and tested with ELISA kits to assess the levels of various immune factor indicators such as IL-10 and macrophage colony stimulating factor (M-CSF).

\subsection{Histopathological examination}

The tissue samples were cut into appropriate size and fixed with $4 \%$ paraformaldehyde for hematoxylineosin staining (H\&E) and immunohistochemical staining. Antibodies used for immunohistochemical analysis were used as follows: F4/80 (Abcam, Cambridge, UK, Cat. \#ab6640), MPO (Abcam, Cambridge, UK, Cat. \#ab208670), TNF-a (Abcam, Cambridge, UK, Cat. \#ab9579). Terminal deoxynucleotidyl transferase-mediated dUTP-biotin nick end labeling assay (TUNEL) was performed for apoptosis.

\subsection{THP-1 cell culture and differentiation into macrophages}

The 1640 complete medium (Gibco, MA, USA) with 1\% double antibody and 10\% FBS (Gibco, MA, USA) were used. THP- 1 cells with ideal growth activity were seeded in six-well plates at a density of $1 \times 10^{6} /$ well, and $100 \mathrm{ng} / \mathrm{mL}$ phorbol 12-myristate 13-acetate (PMA, Sigma, MA,USA, Cat. \#P1585) was added to the medium to induce differentiation for $24 \mathrm{~h}$. Then, the cells were cultured for 4 days, and the morphology of cells was observed under a light microscope. The induced macrophages were collected, and the surface antigens CD11b and CD68 were detected by flow cytometry.

THP-1 cells were induced to differentiate into macrophages and cultured in six-well plates. A 0.4- $\mu \mathrm{m}$ Transwell chamber (Corning, NY) was placed on a six-well plate. Further, $5 \times 10^{5}$ HuMSC-HNF4a were seeded into the chamber (control group with HuMSC-CON and negative control group without cells) and co-cultured with macrophages in 1640 medium for $24 \mathrm{~h}$ to construct co-culture systems. The MTT assay was used to detect the viability of macrophages. The Transwell chamber was discarded after collecting cells of the three groups. The mixed cells were cultured and stimulated in 1640 medium containing LPS $(1 \mu \mathrm{g} / \mathrm{mL})$ for $6 \mathrm{~h}$. The negative control group cultured for another $6 \mathrm{~h}$. The cell culture medium of macrophages in the three groups was collected. Immune factors secreted by macrophages, such as TNF- 
$a$ and IL-1 $\beta$, were detected using ELISA kits. The expression patterns of macrophage CD80 and CD206 were detected by flow cytometry.

\subsection{Statistical analysis}

The data were expressed as mean \pm standard deviation (mean \pm SD). SPSS 19.0 statistical software was used for data analysis. The comparison of indicators depended on a single-factor analysis of variance. The $t$-test was used for comparison between groups. A $P$ value $<0.05$ indicated a statistically difference .

\section{Results}

\subsection{Identification of HuMSCs and HuMSC-HNF4a}

The cells isolated from the umbilical cord were fibroblast-like and grew in the form of fusiform strips adherently under the light microscope (Fig. 1a). The morphology of the cells changed after induction of adipogenesis, osteogenesis, and chondrogenesis. The induced cells were detected using Oil Red $O$ staining, Alizarin red, and type II collagen, and their morphology was found to be in accordance with their differentiation performance (Fig. 1b-1 d). Flow cytometry revealed that the cells overexpressed CD73, CD90, and CD105, but expressed CD34, CD45, and HLA-DR at a low level (Fig. 1e). In summary, the cells met the identification criteria of MSCs [22].

HuMSCs were transfected with either a pWIPI-Green Fluorescent Protein (GFP, HuMSC-CON) or pWIPIHNF4a-GFP (HuMSC-HNF4a) lentiviral vector for 5-7 days. After infection, the ratio of GFP-positive cells revealed that the transfection efficiency was $>95 \%$ (Fig. 2a). Western blot analysis and real-time PCR indicated the obvious upregulation of the expression of HNF4a in HuMSC-HNF4a cells (Fig. 2b). In summary, HuMSC-HNF4a and HuMSC-CON were successfully constructed.

\subsection{Protective effects of HuMSC-HNF4a in mice with ALF}

A survival rate experiment was performed, liver function indexes and the levels of inflammatory factor in serum were detected, and H\&E and immunohistochemical staining were performed after LPS/D-GaIN injection to determine the impact of HuMSC-HNF4a on survival.

The survival curves indicated that the intraperitoneal injection of both HuMSC-HNF4a and HuMSC-CON could improve the survival of mice with ALF $(P<0.05)$ (Fig. 3a). However, the effect of the injection of HuMSC-HNF4a was better. In detail, the mortality in the HuMSC-HNF4a and HuMSC-CON groups after $9 \mathrm{~h}$ was $44 \%$ and $90 \%$, respectively.

The plasma levels of liver enzyme indexes ALT and AST detected in the LPS/D-GalN-induced ALF mouse model were the lowest in the HuMSC-HNF4a group compared with both the HuMSC-CON and PBS groups $(P<0.01)$ (Fig. 3b). 
The levels of pro-inflammatory cytokines in circulation were examined. TNF- $\alpha$ and IL-1 $\beta$ plasma levels were both significantly reduced in the HuMSC-HNF4a and HuMSC-CON groups after ALF. However, the levels of TNF- $\alpha$ and IL-1 $\beta$ in the HuMSC-HNF4a group were lower (Fig. 3c).

H\&E staining revealed extensive congestion and necrosis of liver tissues in the PBS group. The liver tissues in the HuMSC-CON group exhibited moderate microscopic deterioration. In contrast, only mild liver injury was seen in the HuMSC-HNF4a group (Fig. 3d). HuMSC-HNF4a alleviated liver damage in mice with ALF. The number of TUNEL-positive cells in the liver tissues significantly inreased in the PBS group compared with the other two groups. The mice injected with HuMSC- HNF4a showed the lowest level of apoptosis (Fig. 4a).

Next, the study investigated the hepatic infiltration of immune cells by immunohistochemical staining of MPO (a marker of neutrophils), F4/80 (a marker of macrophages), and TNF-a (a marker of liver cell death) [23-25]. Liver sections from the HuMSC-HNF4a group contained fewer F4/80-positive cells (macrophages and Kupffer cells) compared with the HuMSC-CON group. A large number of macrophages infiltrated into liver tissues of mice injected with PBS (Fig. 4b). TNF-a, secreted by activated macrophages, is the most important inflammatory factor that causes hepatocyte apoptosis and necrosis during the pathogenesis of ALF [23]. The level of TNF-a in liver tissues of mice injected with HuMSCHNF4a was lower than that in the HuMSC-CON group (Fig. 4b). No significant difference in neutrophil infiltration in liver tissues was observed among these three groups (Fig. 4b). Taken together, these results indicated that HuMSC-HNF4a mainly acted on macrophages. Experiments were subsequently designed to explore specific mechanism of action of macrophages action in vitro.

\subsection{HuMSC-HNF4a inhibited LPS-stimulated macrophage inflammation and promoted macrophages to polarize to M2 states}

Circular and suspended THP-1 cells were cultured with PMA $(100 \mathrm{ng} / \mathrm{ml})$ for $24 \mathrm{~h}$; the cells grew adherently and lost the ability to proliferate subsequently. After incubation for 4 days, the cells became larger and developed extended amoeba-like pseudopodia (Fig. 5a). The expression of human macrophage markers CD11b and CD68 was significantly higher in differentiated cells compared with undifferentiated cells (Fig. 5b). In summary, the cells met the criteria of experimental macrophages [26].

Macrophages co-cultured with HuMSC-HNF4a or HuMSC-CON were stimulated with LPS for $6 \mathrm{~h}$. The cultured supernatant was collected, and the levels of TNF- $\alpha$ and IL-1 $\beta$ were determined. Macrophages released a large number of inflammatory factors, such as TNF- $\alpha$ and IL-1 $\beta$, after stimulation. Either HuMSC-HNF4a or HuMSC-CON decreased the levels of inflammatory factors secreted by macrophages. HuMSC-HNF4a inhibited the release of TNF-a more significantly $(P<0.01)$. No difference was found in the effect of inhibiting the release of IL-1 $\beta$ between HuMSC-HNF4a and HuMSC-CON ( Fig. 5c).

Macrophages polarized towards M1 phenotype or M2 phenotype according to the local microenvironment, which played a key role in the development of ALF. M1 macrophages were characterized by the overexpression of CD80, CD86, and so forth. M2 penotype overexpressed CD206, 
CD369 (Dectin-1), and so forth [27, 28]. The expression of CD80 and CD206 was examined by flow cytometry after collecting macrophages co-cultured with HuMSC-HNF4a or HuMSC-CON cells stimulated with LPS for $6 \mathrm{~h}$ and macrophages without LPS (CTR). The expression of CD80 in macrophages cocultured with HuMSC-HNF4a decreased. In contrast, the expression of CD206 increased (Fig. 5d). Overall, these results indicated that HuMSC-HNF4a promoted M2 polarization of macrophages, which was consistent with previous conclusions that HuMSC-HNF4a reduced the level of TNF-a secreted by macrophages.

\subsection{Overexpression of HNF4a up-regulated the level of IL-10 and M-CSF secreted by HuMSCs}

The supernatants in the HuMSC-HNF4a and HuMSC-CON groups were collected for protein chip analysis to determine the mechanism of HuMSC-HNF4a in regulating macrophage polarization (Fig. 6a). The results showed that the overexpression of HNF4a changed the levels of IL-10 and M-CSF. The protein-chip assay was performed to verify the results, and the same conclusion was obtained (Fig.6b). The mice were pretreated with HuMSC-CON and HuMSC-HNF4a for 24h as previously described, and an ALF model was constructed to elucidate further how HuMSC-HNF4a protected mice from ALF. The liver, spleen, lung, and small intestine of mice in these two groups were collected to detect GFP fluorescence. The results indicated no distribution of HuMSCs in these organs (Fig. 6c). The difference in serum IL-10 and M-CSF cells suggested that HuMSC-HNF4a cells reduced ALF in mice via the release of IL-10 and M-CSF, independent of their locations in the liver (Fig. 6d). The percentages of M1 and M2 phenotypes of macrophages in the liver after cell input therapy were observed by flow cytometry to determine whether the overexpression of HNF4a in HuMSCs could affect macrophage polarization in vivo (Fig. 7a). HuMSCHNF4a promoted macrophages to polarize to M2 states in vivo. In addition, recent studies confirmed that IL-10 and M-CSF promoted the polarization of liver macrophages (Kupffer cells) toward the M2 phenotype and play a role in inhibiting inflammation and repairing damage $[29,30]$. It was inferred that HNF4a might enhance the immunoregulatory function of HuMSCs by increasing the secretion of IL-10 and M-CSF.

\section{Discussion}

The use of genetically modified cells, with more obvious therapeutic effects, has replaced the use of existing cells to treat diseases with the rapid development of genetic engineering technology. Hence, attempts were made to use gene-regulated MSC transplantation for treating ALF and hence enhance the therapeutic effect of MSCs.

Previous studies showed that the overexpression of HNF4a in HuMSCs promoted HuMSC differentiation into hepatocytes $[10,14]$. They also demonstrated that HNF4a, with strong regulatory effects on various functions of MSCs, was closely related to ALF. Thus, it was suggested that HuMSCs with the overexpression of HNF4 might possess amazing therapeutic effects on ALF.

Therefore, a series of experiments were performed to verify this hypothesis. HuMSC-HNF4a effectively improved the survival of mice with ALF, suppressed the immune responses, reduced liver damage, and 
reduced necrosis and apoptosis of liver tissues in vivo. The therapeutic effect of HuMSC-HNF4a cells on ALF was to inhibit the inflammatory response of liver macrophages, reduce the infiltration of macrophages in liver tissues, and enhance the release of inflammatory factors.

Based on the importance of macrophages in the pathogenesis of ALF, an experiment was designed in this study to co-culture HuMSC-HNF4a and macrophages so as to explore the effect of HuMSC-HNF4a on the inflammatory response of macrophages in vitro. HuMSC-HNF4a inhibited the inflammatory responses caused by LPS stimulation and reduced the level of TNF-a secreted by macrophages. In vivo, experiments were designed to testify that HuMSC-HNF4a could promote macrophage polarization to the M2 phenotype by increasing the secretion of IL-10 and M-CSF, thereby reducing liver injury in mice.

HuMSC-HNF4a promoted macrophage polarization toward the M2 phenotype, suggesting that HuMSCHNF4a changed the microenvironment of the culture medium. The culture supernatants of HuMSCHNF4a and HuMSC-CON were collected for protein chip analysis to examine the distinguishing factors so as to find the factors secreted by HuMSC-HNF4a influencing the polarization of macrophages. More than 40 factors with significance were found $(P<0.05)$. The factors related to the inflammatory response of macrophage were examined to confirm that the levels of IL-10 and M-CSF secreted by HuMSC-HNF4a were significantly higher than the levels of those secreted by HuMSC-CON. IL-10 and M-CSF activated the polarization of macrophages toward the M2 phenotype, inhibited inflammation, and repaired damage [29, 30]. Thus, it was speculated that HNF4a enhanced the immune-regulatory function of HuMSCs by increasing the secretion of immune factors such as IL-10 and M-CSF.

Recent studies confirmed that immunoinflammatory responses were important in ALF. Hence, the immunomodulatory function of MSCs has become the latest focus. However, several studies have shown that hepatic macrophages, as the key cells in inflammation regulation, are central in the pathogenesis of liver injury and have been proposed as the potential targets in combatting fibrosis [31]. The last few years have revealed several key regulators of macrophage polarization, including the signal transducer and activator of transcription family of molecules such as macrophage chemoattractant CCL2 [32], peroxisome proliferator-activated receptor gamma [33], CCAAT inhancer-binding proteins (C/EBP) family, and interferon regulatory factors [27]. Recent studies have also suggested that microRNAs and long noncoding RNAs influence macrophage polarization [34]. The present study reported another regulatory mechanism of macrophage polarization. MSCs exerted immunoregulatory functions on lymphocytes, NK T cells, DC cells, and other immune effector cells in ALF caused by different etiologies. Similarly, the study also found that HuMSC-HNF4a had a stronger anti-inflammatory effect on macrophages in ALF compared with HuMSCs used alone. This finding indicated that the effects of both MSCs and HNF4a were complicated. An unknown mechanism might exist, which needs exploration.

HuMSC-HNF4a with a powerful immunomodulatory effect in the pathogenesis of ALF is expected to form a mixed system with immortalized hepatocytes to jointly transplant or construct a hybrid biological artificial liver. This process combines functional support and immune regulation to treat ALF more effectively and regarded as a future research direction. However, this study also had certain limitations. 
After the protein-chip assay, the mechanism of HNF4a gene regulating the secretion of immune factors by MSCs needs to be further explored in vivo. In addtiton, database analysis was performed to explore the upstream transcription factors related to the differencial gene expression according to the sequencing in ChIP-X Enrichment Analysis Version 3 (ChEA3). The bar chart revealed the relevance between HNF4a and the differencial gene including IL-10 and M-CSF囚Fig. S1). Of course, the possible mechanism needs further verification.

\section{Conclusion}

The present study confirmed the therapeutic effects of HuMSC-HNF4a in ALF in vivo and in vitro. It revealed that HuMSC-HNF4a cells promoted macrophages to polarize towards the M2 phenotype by secreting IL-10 and M-CSF. HuMSC-HNF4a suppressed inflammation and repaired damage, which actively interfered with excessive inflammatory reactions in the body instead of passive support. Meanwhile, the findings provided new ideas for scientific research and clinic practice.

\section{Abbreviations}

ALF, Acute liver failure; D-GaIN, D-galactosamine; HNF4a, hepatocyte nuclear factor 4a; HuMSC-HNF4a, HuMSCs overexpressing HNF4a; HuMSCs, human umbilical cord mesenchymal stem cells; HuMSC-CON, HuMSCs without HNF4a; LPS, lipopolysaccharide; MSCs, mesenchymal stem cells; IL-10, interleukin-10; M-CSF, macrophage colony stimulating factor; AST, aspartate aminotransferase; ALT, alanine transaminase

\section{Declarations}

\section{Acknowledgements}

Not applicable.

\section{Authors' contributions}

YPY, QQZ, QX, and HHL were involved in conception and design of the study. NW and QQZ performed the in vivo experiment. YPY and QQZ performed the in vitro experiments. JC and LX performed the data analysis and interpretation. YPY, JJZ, and HLH wrote the manuscript. NW, QX, JZ, JJZ, and HLH were involved in review and editing of the manuscript. All authors commented the final version. All authors had full access to all data. All authors read and approved the final manuscript.

\section{Funding}

This study was supported by the National Natural Science Foundation of China (81570561 to HL Hang), the Shanghai Pujiang Program (17PJD023 to HL Hang), the Shanghai Municipal Education Commission- 
Gaofeng Clinical Medicine Grant Support (20181806 to HL Hang), and the Most Important Clinical Medical Center in Shanghai (2017ZZ01018 to Q Xia).

\section{Availability of data and materials}

All data and materials used in this work are available on request.

\section{Ethics approval and consent to participate}

This study was approved by the Institutional Review Board and Human Ethics Committee of Ren Ji Hospital, School of Medicine, Shanghai Jiao Tong University, Shanghai, China.

\section{Consent for publication}

All authors consent to publish this manuscript.

\section{Competing interests}

The authors declare that they have no competing interests.

\section{Author details}

${ }^{1}$ Department of Liver Surgery, RenJi Hospital, School of Medicine, Shanghai Jiao Tong University, Shanghai, China

${ }^{2}$ Department of Hepatopancreatobiliary Surgery, East Hospital Affiliated to Tongji University, Shanghai 200123, China.

\section{References}

1. Bernal W, Lee WM, Wendon J, Larsen FS, Williams R. Acute liver failure: A curable disease by 2024 ? J Hepatol. 2015;62:S112-20.

2. Wendon J, Cordoba J, Dhawan A, Larsen FS, Manns M, Samuel D, et al. EASL Clinical Practical Guidelines on the management of acute (fulminant) liver failure. J Hepatol. 2017;66:1047-81.

3. Wang YH, Wu DB, Chen B, Chen EQ, Tang H. Progress in mesenchymal stem cell-based therapy for acute liver failure. Stem Cell Res Ther. 2018;9:227.

4. Liang H, Huang K, Su T, Li Z, Hu S, Dinh PU, et al. Mesenchymal Stem Cell/Red Blood Cell-Inspired Nanoparticle Therapy in Mice with Carbon Tetrachloride-Induced Acute Liver Failure. ACS Nano. 2018;12:6536-44.

5. Lee CW, Chen YF, Wu HH, Lee OK. Historical Perspectives and Advances in Mesenchymal Stem Cell Research for the Treatment of Liver Diseases. Gastroenterology. 2018;154:46-56.

6. Ahmadi AR, Chicco M, Wesson RN, Anders RA, Dor F, JNM IJ, et al. Stem Cell Mobilization Is Lifesaving in a Large Animal Preclinical Model of Acute Liver Failure. Ann Surg. 2018;268:620-31. 
7. Chen YF, Tseng CY, Wang HW, Kuo HC, Yang VW, Lee OK. Rapid generation of mature hepatocyte-like cells from human induced pluripotent stem cells by an efficient three-step protocol. Hepatology. 2012;55:1193-203.

8. Torrado EF, Gomes C, Santos G, Fernandes A, Brites D, Falcão AS. Directing mouse embryonic neurosphere differentiation toward an enriched neuronal population. International journal of developmental neuroscience : the official journal of the International Society for Developmental Neuroscience. 2014;37:94-99.

9. Hu J, Yang Z, Wang J, Tang Y, Liu H, Zhang B, et al. Infusion of Trx-1-overexpressing hucMSC prolongs the survival of acutely irradiated NOD/SCID mice by decreasing excessive inflammatory injury. PloS one. 2013;8:e78227.

10. Seo K-W, Sohn S-Y, Bhang D-H, Nam M-J, Lee H-W, Youn H-Y. Therapeutic effects of hepatocyte growth factor-overexpressing human umbilical cord blood-derived mesenchymal stem cells on liver fibrosis in rats. Cell biology international. 2014;38:106-16.

11. Lau HH, Ng NHJ, Loo LSW, Jasmen JB, Teo AKK. The molecular functions of hepatocyte nuclear factors - In and beyond the liver. Journal of hepatology. 2018;68:1033-48.

12. Lu H. Crosstalk of HNF4 with extracellular and intracellular signaling pathways in the regulation of hepatic metabolism of drugs and lipids. Acta pharmaceutica Sinica. B. 2016;6:393-408.

13. Hang H, Yu Y, Wu N, Huang Q, Xia Q, Bian J. Induction of highly functional hepatocytes from human umbilical cord mesenchymal stem cells by HNF4alpha transduction. PLoS One. 2014;9:e104133.

14. Wu N, Zhang Y-L, Wang H-T, Li D-W, Dai H-J, Zhang Q-Q, et al. Overexpression of hepatocyte nuclear factor $4 \mathrm{a}$ in human mesenchymal stem cells suppresses hepatocellular carcinoma development through Wnt/ $\beta$-catenin signaling pathway downregulation. Cancer biology \& therapy. 2016;17:55865.

15. Li C, Xu MM, Wang K, Adler AJ, Vella AT, Zhou B. Macrophage polarization and meta-inflammation. Translational research : the journal of laboratory and clinical medicine. 2018;191:29-44.

16. Sica A, Invernizzi P, Mantovani A. Macrophage plasticity and polarization in liver homeostasis and pathology. Hepatology. 2014;59:2034-42.

17. Funes SC, Rios M, Escobar-Vera J, Kalergis AM. Implications of macrophage polarization in autoimmunity. Immunology. 2018;154:186-95.

18. Atri C, Guerfali FZ, Laouini D. Role of Human Macrophage Polarization in Inflammation during Infectious Diseases. Int J Mol Sci. 2018;19.

19. Vasandan AB, Jahnavi S, Shashank C, Prasad P, Kumar A, Prasanna SJ. Human Mesenchymal stem cells program macrophage plasticity by altering their metabolic status via a PGE2-dependent mechanism. Sci Rep. 2016;6:38308.

20. Philipp D, Suhr L, Wahlers T, Choi YH, Paunel-Gorgulu A. Preconditioning of bone marrow-derived mesenchymal stem cells highly strengthens their potential to promote IL-6-dependent M2b polarization. Stem Cell Res Ther. 2018;9:286. 
21. François M, Romieu-Mourez R, Li M, Galipeau J. Human MSC suppression correlates with cytokine induction of indoleamine 2,3-dioxygenase and bystander M2 macrophage differentiation. Molecular therapy : the journal of the American Society of Gene Therapy. 2012;20:187-95.

22. Du Z, Wei C, Yan J, Han B, Zhang M, Peng C, et al. Mesenchymal stem cells overexpressing C-X-C chemokine receptor type 4 improve early liver regeneration of small-for-size liver grafts. Liver Transpl. 2013;19:215-25.

23. Eguchi A, Wree A, Feldstein AE. Biomarkers of liver cell death. Journal of hepatology. 2014;60:106374.

24. Austyn JM, Gordon S. F4/80, a monoclonal antibody directed specifically against the mouse macrophage. European journal of immunology. 1981;11:805-15.

25. Aratani Y. Myeloperoxidase: Its role for host defense, inflammation, and neutrophil function. Archives of biochemistry and biophysics. 2018;640:47-52.

26. Saylor J, Ma Z, Goodridge HS, Huang F, Cress AE, Pandol SJ, et al. Spatial Mapping of Myeloid Cells and Macrophages by Multiplexed Tissue Staining. Frontiers in immunology. 2018;9:2925.

27. Sica A, Invernizzi P, Mantovani A. Macrophage plasticity and polarization in liver homeostasis and pathology. Hepatology (Baltimore, Md.). 2014;59:2034-42.

28. Magatti M, Vertua E, De Munari S, Caro M, Caruso M, Silini A, et al. Human amnion favours tissue repair by inducing the M1-to-M2 switch and enhancing M2 macrophage features. Journal of tissue engineering and regenerative medicine. 2017;11:2895-911.

29. Chung S, Ranjan R, Lee YG, Park GY, Karpurapu M, Deng J, et al. Distinct role of FoxO1 in M-CSF- and GM-CSF-differentiated macrophages contributes LPS-mediated IL-10: implication in hyperglycemia. Journal of leukocyte biology. 2015;97:327-39.

30. Makita N, Hizukuri Y, Yamashiro K, Murakawa M, Hayashi Y. IL-10 enhances the phenotype of M2 macrophages induced by IL-4 and confers the ability to increase eosinophil migration. International immunology. 2015;27:131-41.

31. Tacke F, Zimmermann HW. Macrophage heterogeneity in liver injury and fibrosis. Journal of hepatology. 2014;60:1090-96.

32. Oh DY, Morinaga H, Talukdar S, Bae EJ, Olefsky JM. Increased macrophage migration into adipose tissue in obese mice. Diabetes. 2012;61:346-54.

33. Sica A, Mantovani A. Macrophage plasticity and polarization: in vivo veritas. The Journal of clinical investigation. 2012;122:787-95.

34. Ishii M, Wen $\mathrm{H}$, Corsa CAS, Liu T, Coelho AL, Allen RM, et al. Epigenetic regulation of the alternatively activated macrophage phenotype. Blood. 2009;114:3244-54.

\section{Figures}


$\mathbf{a}$

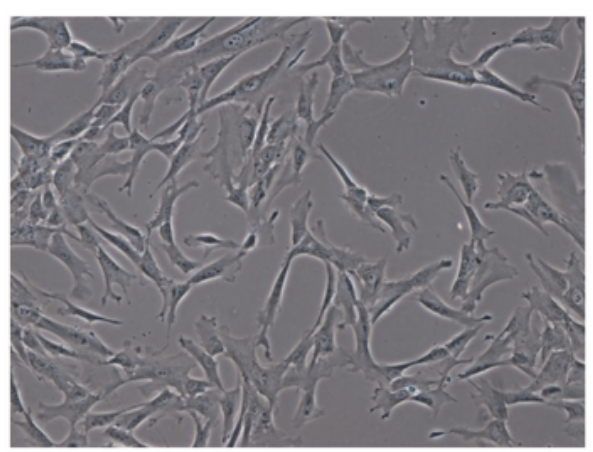

C

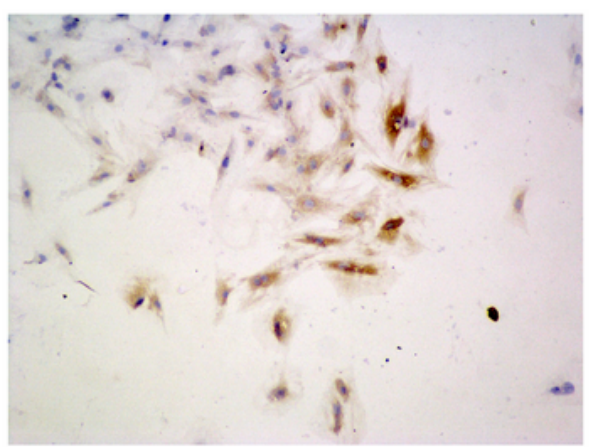

b

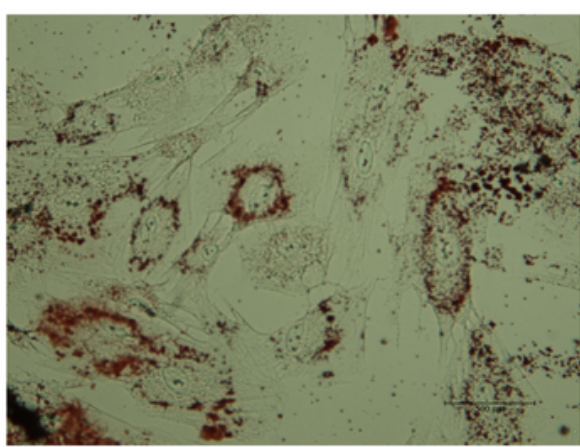

d

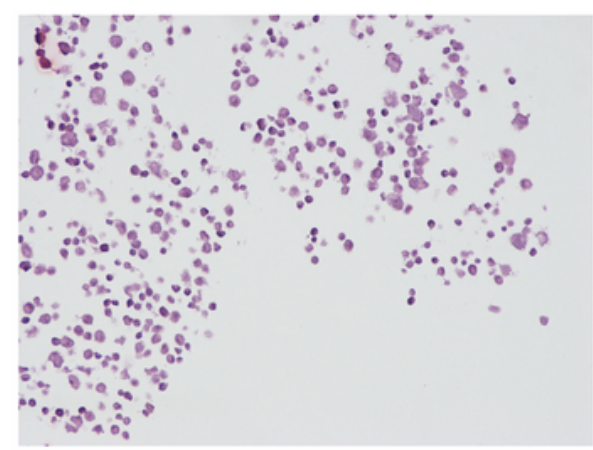

$\mathbf{e}$
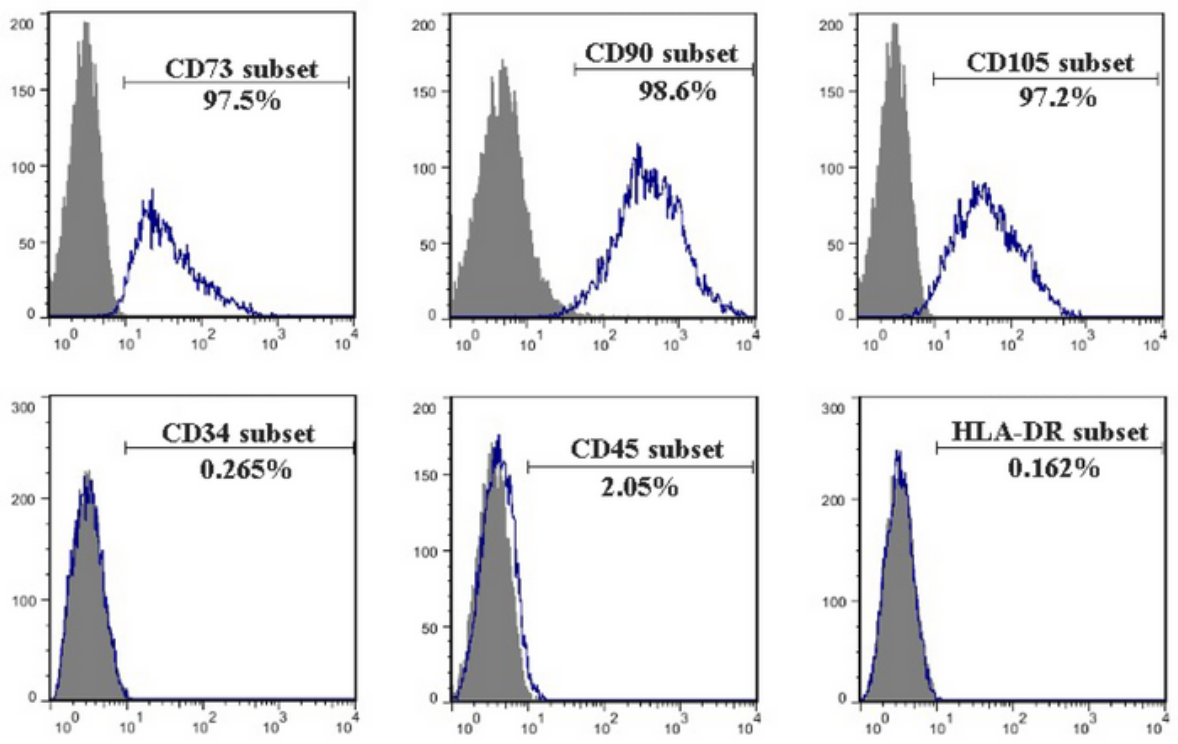

\section{Figure 1}

Identification of HuMSCs. (a) Light microscopic observation of HuMSCs (origin magnification $\times 100)$. (b) Oil red $O$ staining after inducing adipogenic differentiation (origin magnification $\times 100$ ). (c) Alizarin red staining after osteogenic-specific induction (origin magnification $\times 100$ ). (d) Immunohistochemical staining for type II collagen after chondrogenic differentiation conditions (origin magnification $\times 100)$. (e) 
Flow cytometry identification of MSCs: positive for CD73, CD90 and CD105; negative for CD34,CD45 and HLA-DR.

a
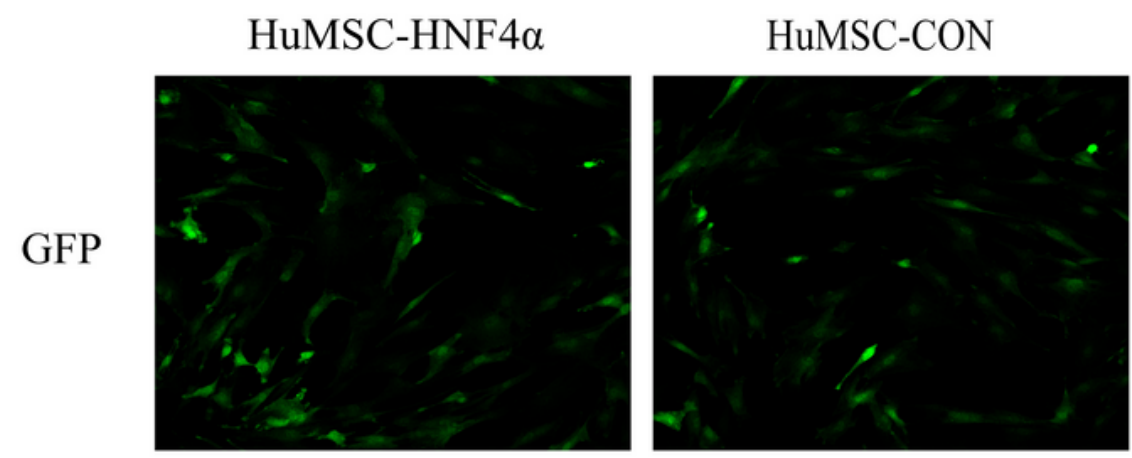

DAPI
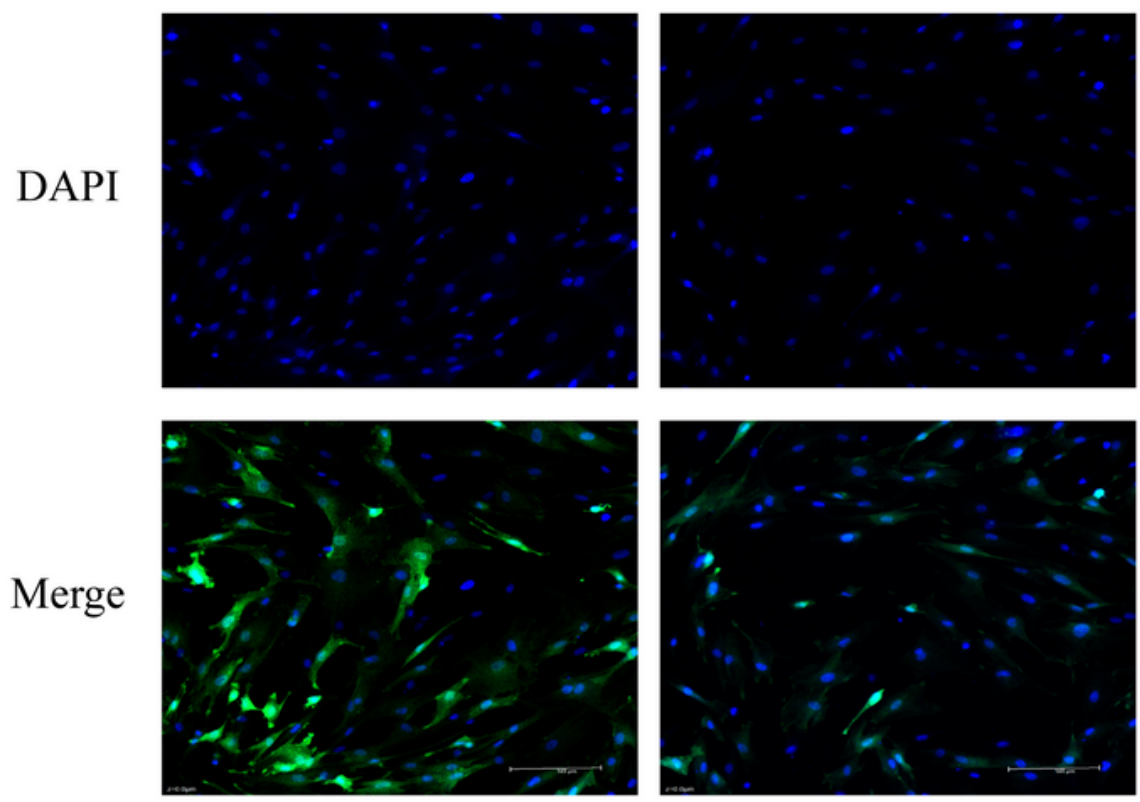

b

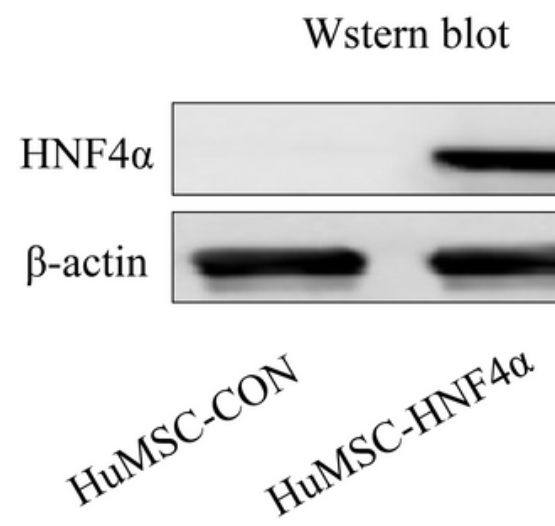

c

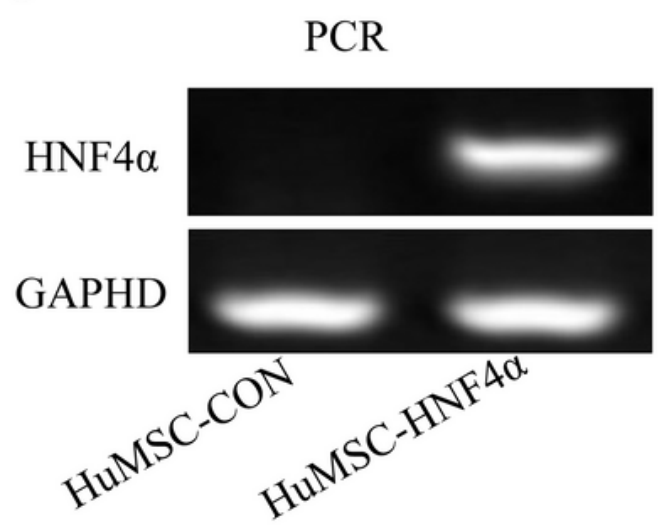

\section{Figure 2}

Establishment and identification of HuMSC-HNF4a. (a) Detection of HNF4a transfection with DAPI nuclear staining, more than 95\% HuMSCs stably expressed GFP. (b) HNF4a protein expression was 
analyzed by western blot. (c) Real-time PCR in HuMSC-HNF4a and HuMSC-CON 5-7 days after lentiviral transfection.

$\mathbf{a}$ Survival after treated by D-Galn/LPS

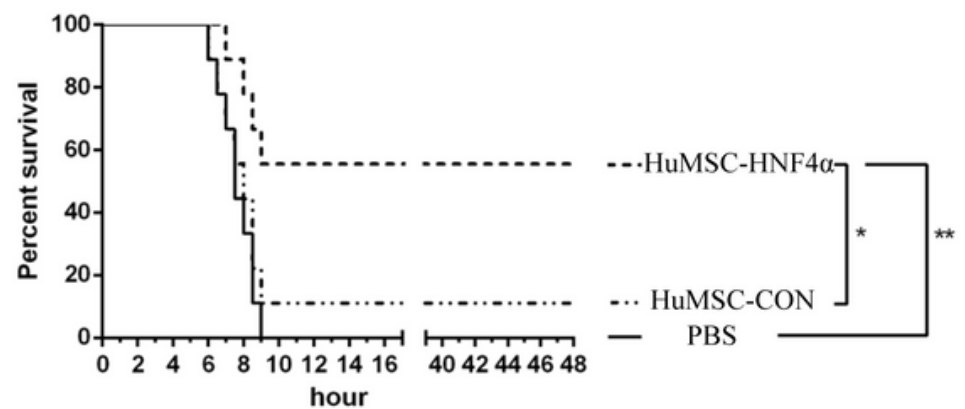

b
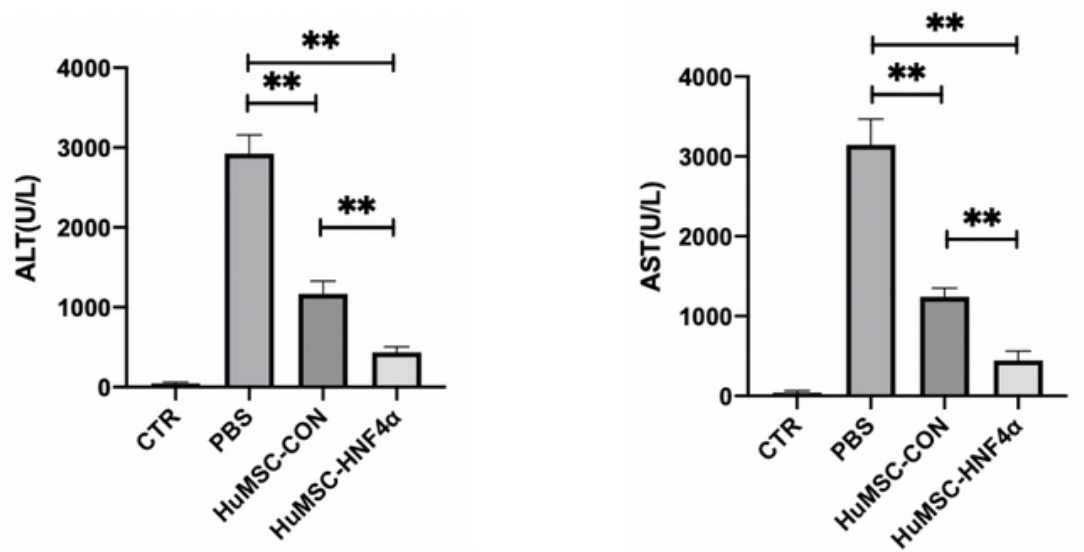

c
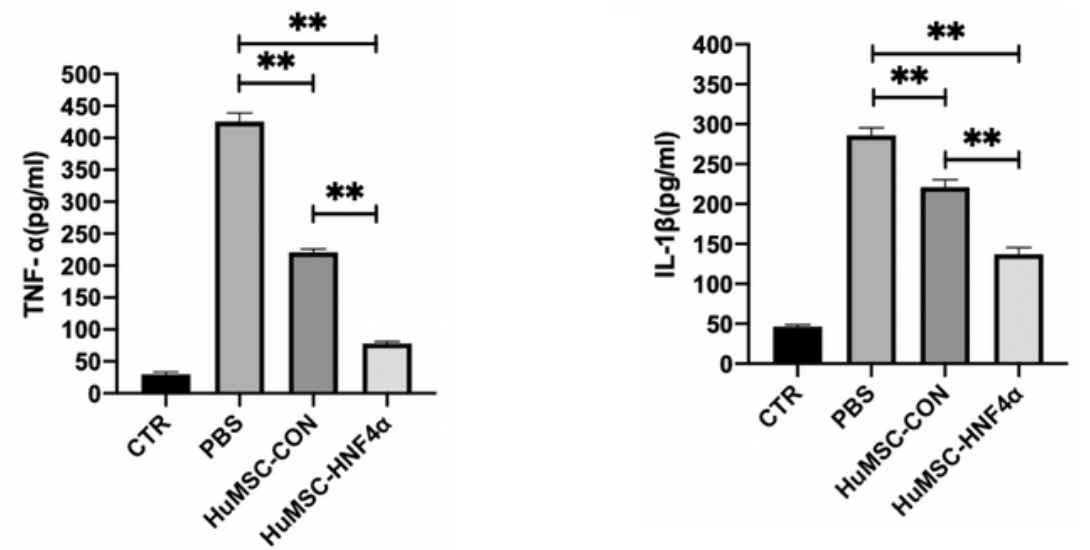

d

\section{PBS}

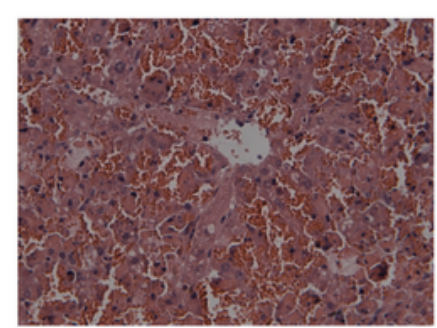

HuMSC-CON

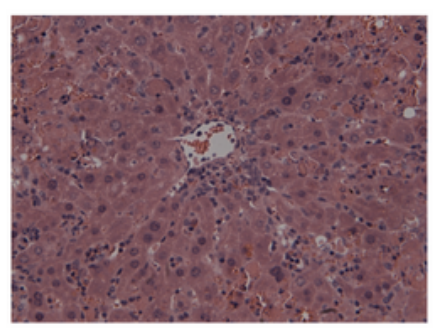

\section{HuMSC-HNF4 $\alpha$}

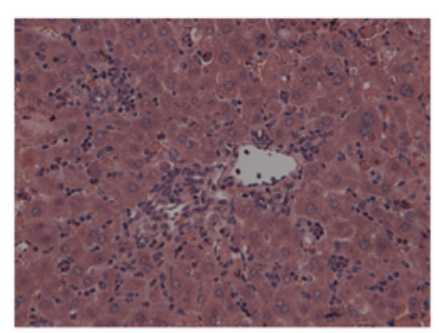

\section{Figure 3}

Protective effects of HuMSC-HNF4a in mice with LPS/D-GalN-induced ALF. (a) Survival curve $(n=10)$. (b) Levels of serum ALT and AST ( $n=4-6)$. (c) Levels of TNF- $\alpha$ and IL-1 $\beta$ in serum ( $n=4-6)$. (d) H\&E stained 
liver tissues in different groups (origin magnification $\times 100, n=3$ ). All data are shown as the mean \pm SD. $* * p<0.01$.

a

PBS

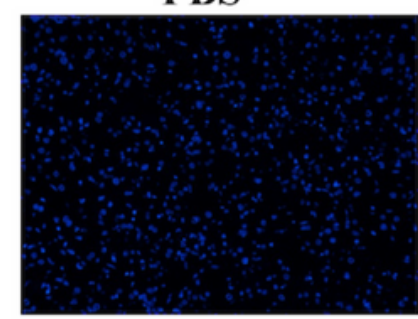

DAPI

TUNEL
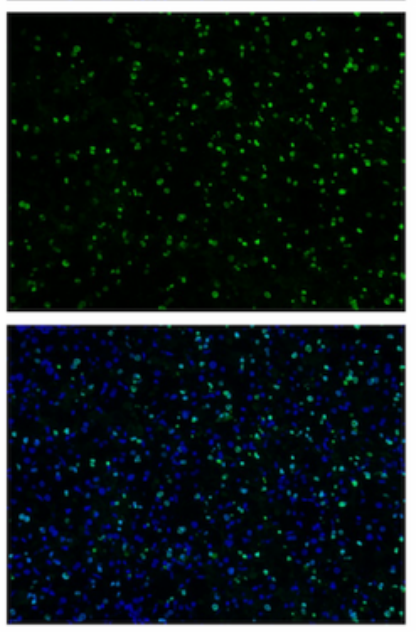

b

F4/80
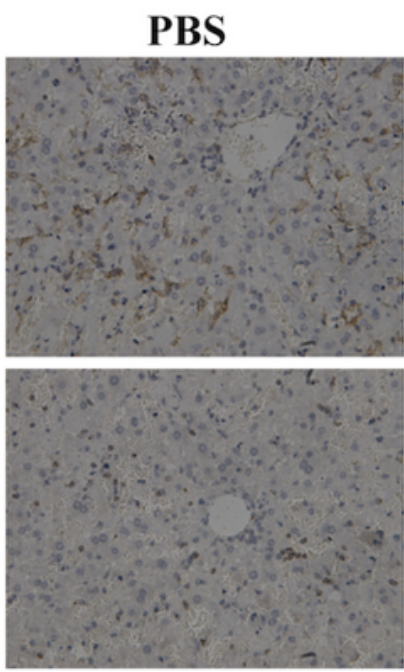

MPO

TNF- $\alpha$

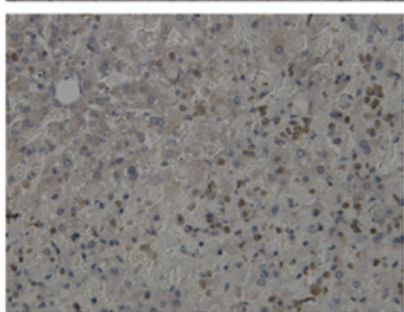

HuMSC-CON
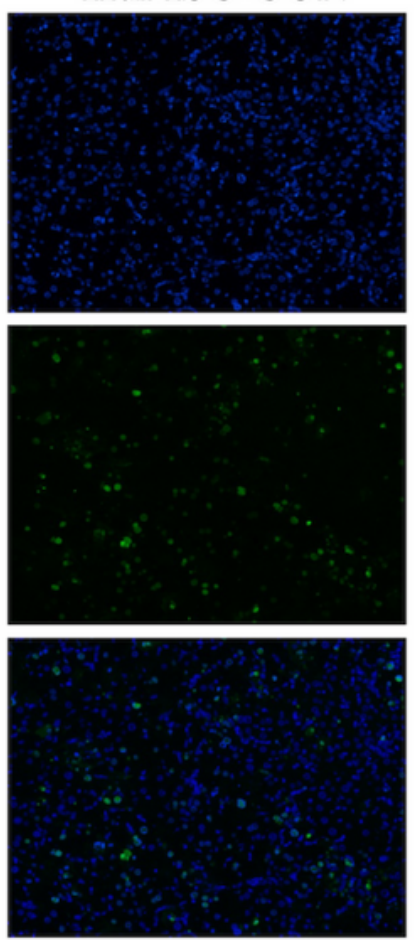

HuMSC-CON
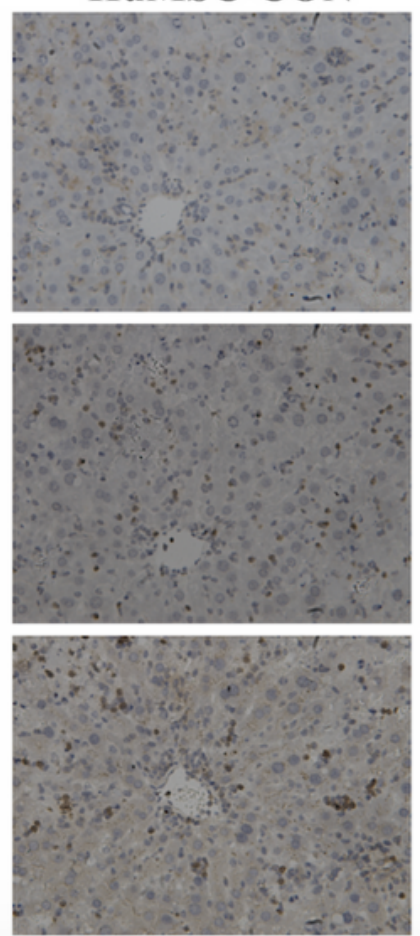

\section{HuMSC-HNF4a}
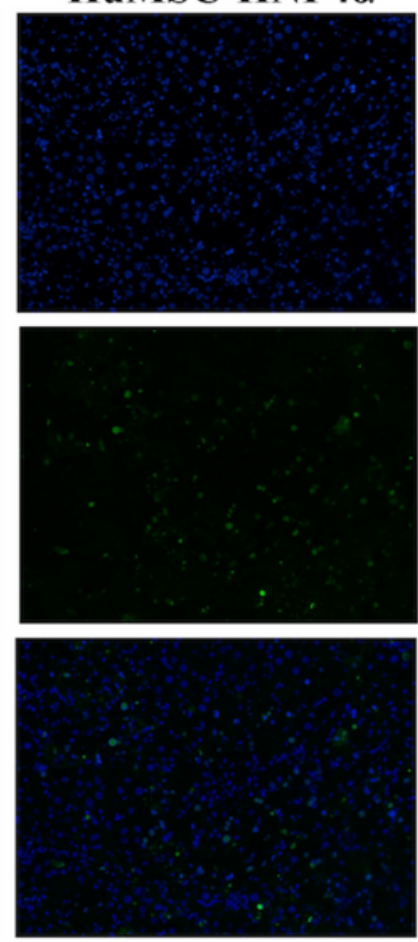

\section{HuMSC-HNF4a}
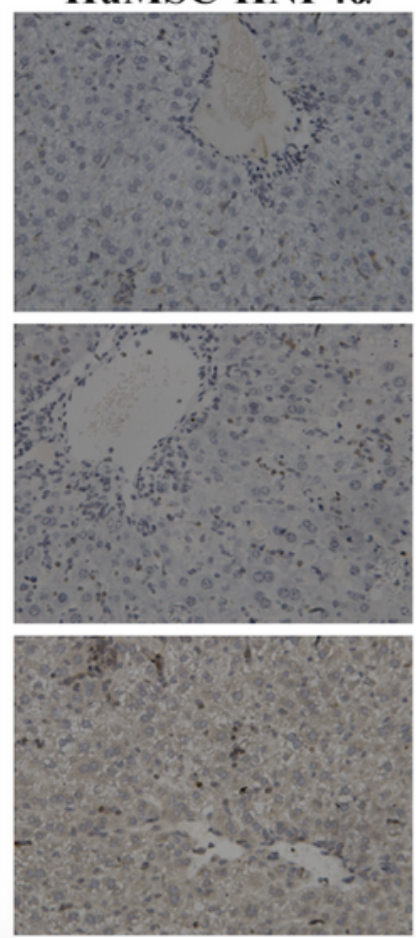

Figure 4

Protective effects of HuMSC-HNF4a on pathology in mice with LPS/D-GalN-induced ALF. (a) Representative fluorescence images of TUNEL staining in liver tissues (origin magnification $\times 100$ ). (b) 
Immunohistochemistry of F4/80, MPO, and TNF-a in the liver with LPS/D-GaIN challenge (origin magnification $\times 100, n=3)$.
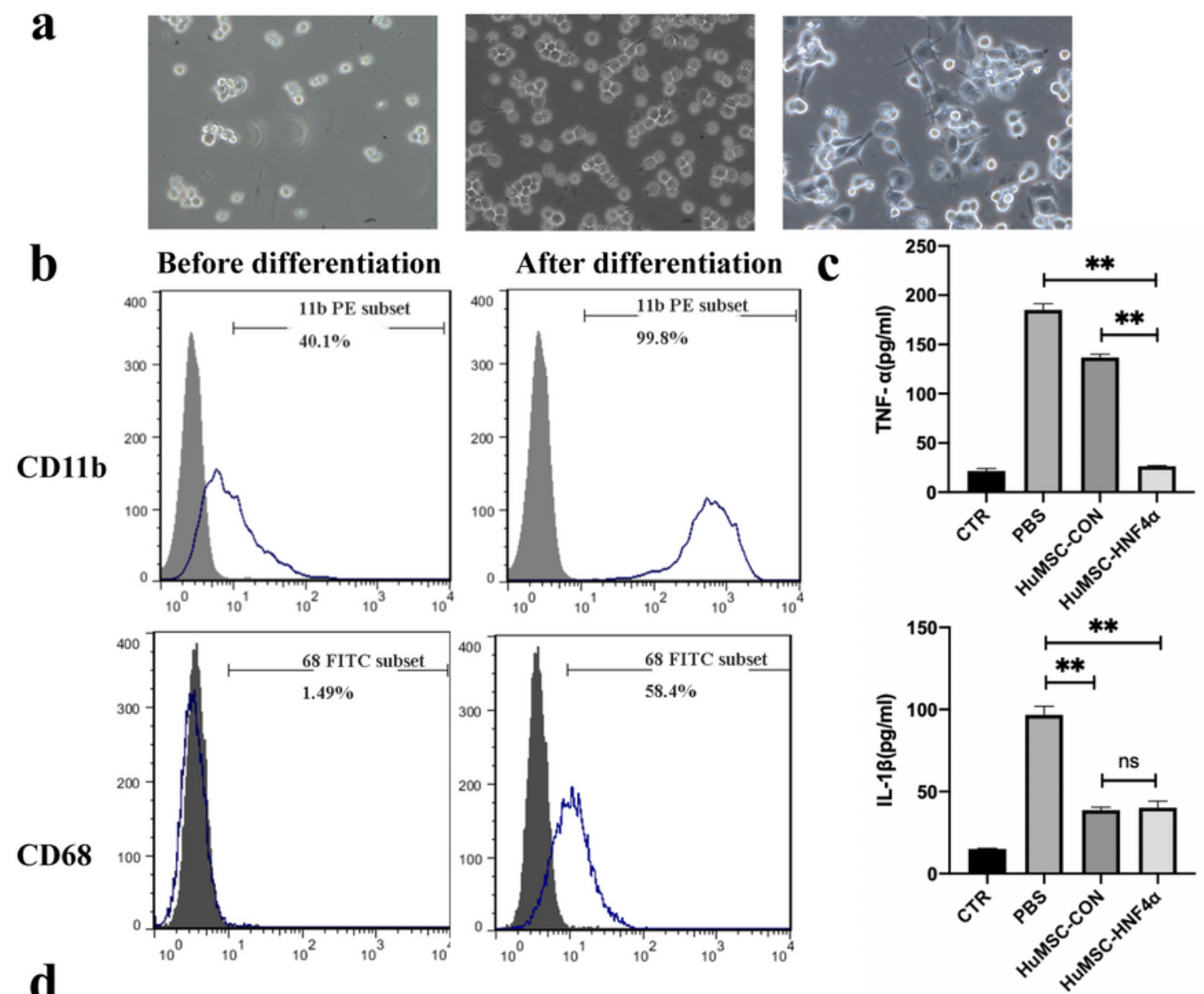

d

LPS
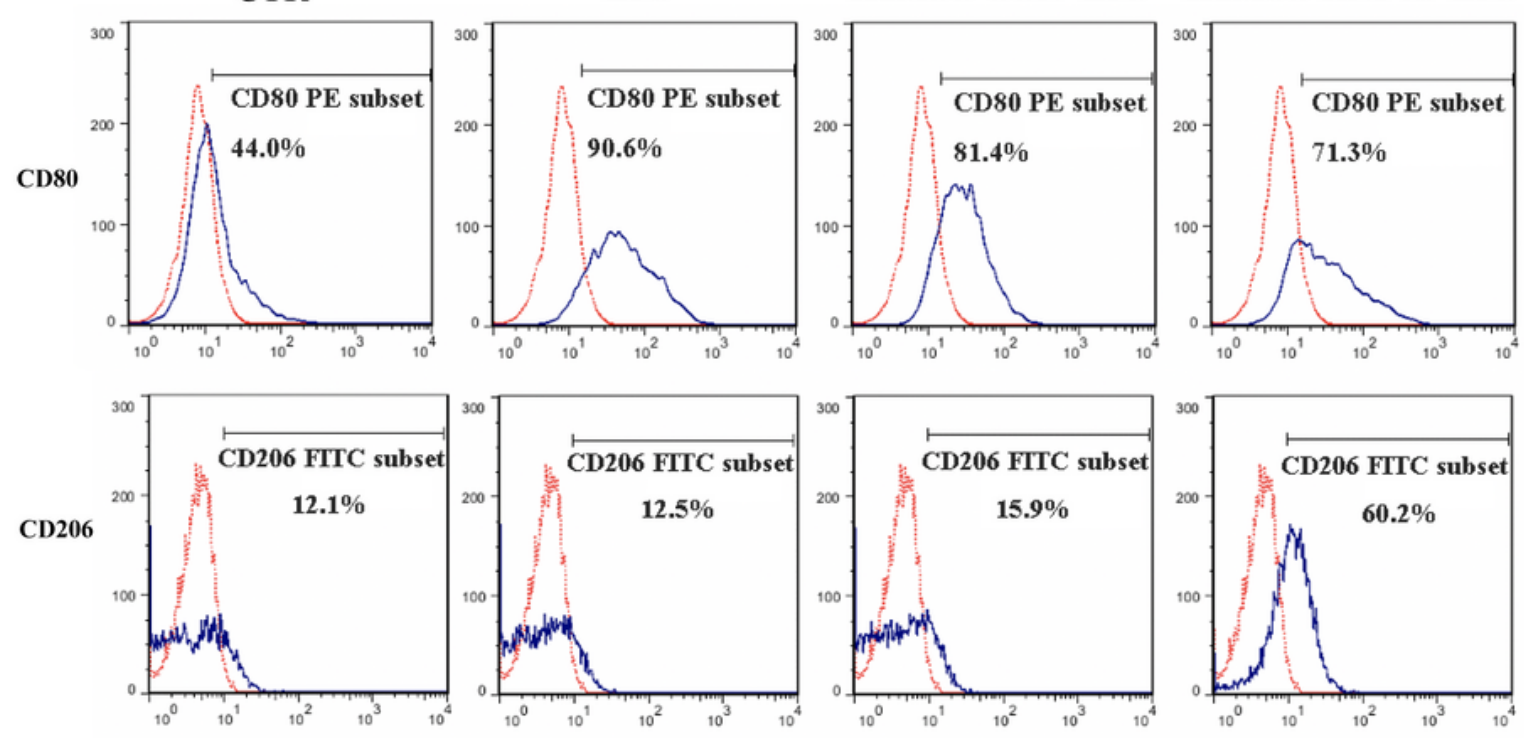

Figure 5

HuMSC-HNF4a inhibited LPS-stimulated macrophage inflammation and promoted macrophage polarization toward the M2 phenotype. (a) Morphological observation of THP-1 cells (origin magnification $\times 100)$. (b) Detection of human macrophage markers (CD11b and CD68) on the surface of 
THP-1 cells by flow cytometry. Macrophages induced by THP-1 cells met the criteria for experimental human macrophages. (c) Levels of TNF- $a$ and IL-1 $\beta$ from the supernatant of the culture medium of each group. (d) Expression of CD80 and CD206 markers of macrophages in each group by flow cytometry. All data are shown as the mean \pm SD. ${ }^{* *} p<0.01$, and ns not significant.

$\mathbf{a}$

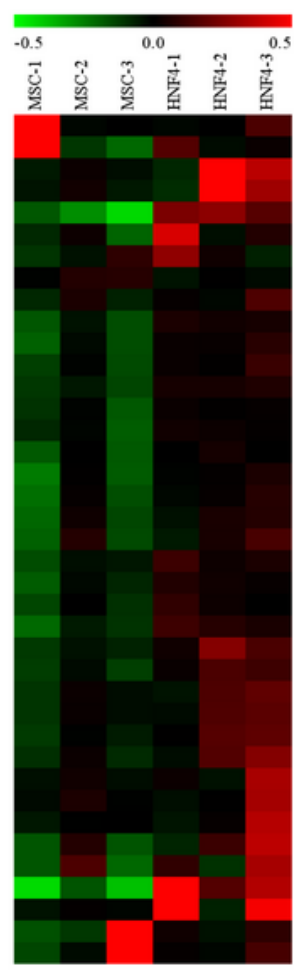

BD-1

PDGF-D
BMP-3b / GDF-10

BMP-3

GCSF

TGF-beta 5

ErbB2

AR (Amphiregulin)

IGF-I SR

IGF-1 SR
IGF-II
CCL14 / HCC-1 / HCC-3

FGF-8

FGF-5

L-31 RA

IL-1 alpha

GF-II R

L-1 beta

L-10

SFRP-1

SFRP-3

IL-17

MCP-2

GF-10 / KGF-2

$\mathrm{MCP}-4 / \mathrm{CCL} 13$

$\mathrm{M}-\mathrm{CSF}$

L-10 R beta

Angiopoietin-like 1

Angiopoietin-4

BMP-6

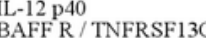

IL-8

CCL28 / VIC

CCR1

d
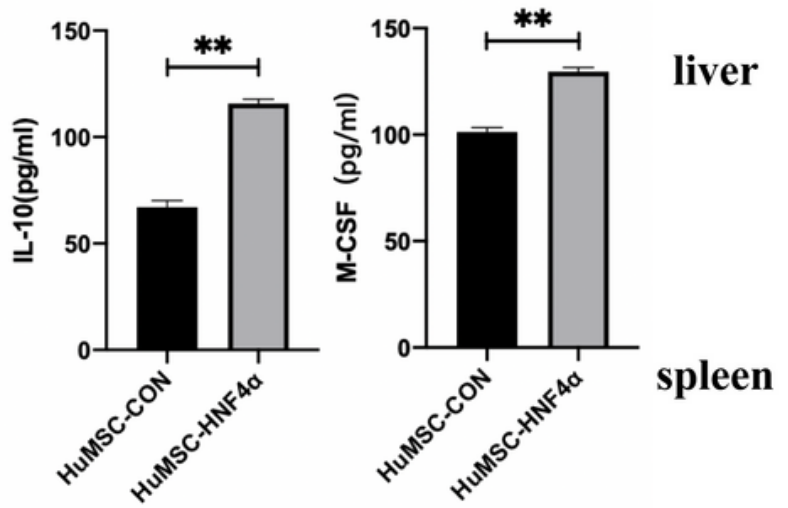

b
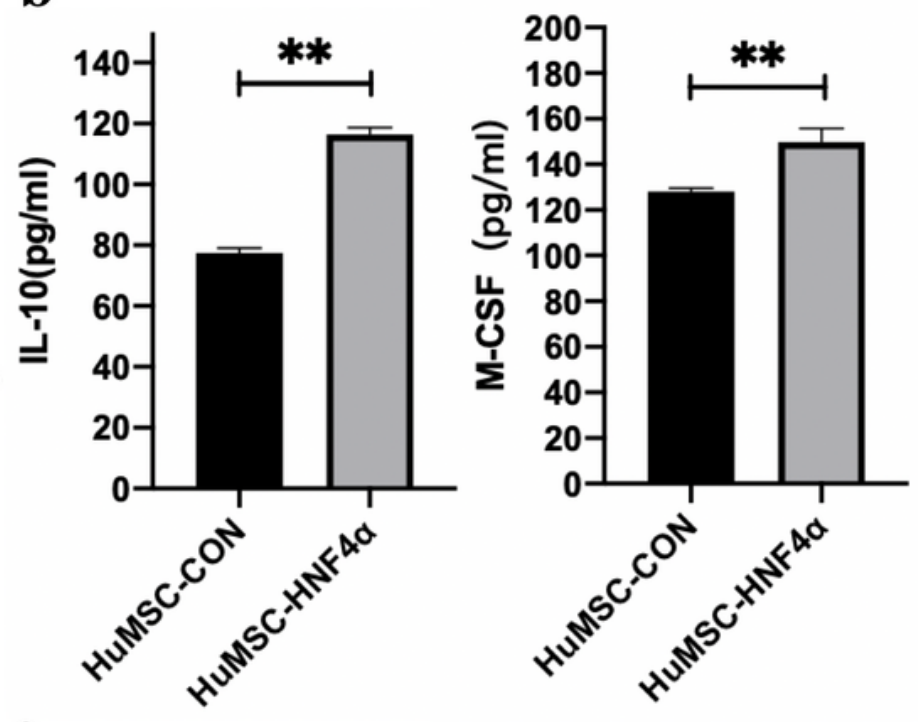

intestine
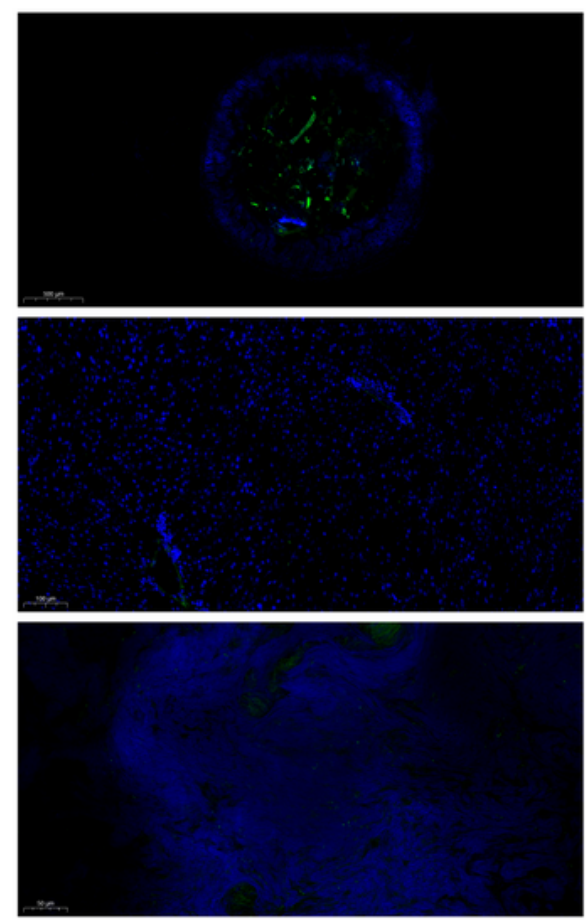

lung

Figure 6 
Overexpression of HNF4a upregulated the level of IL-10 and M-CSF secreted by HuMSCs. (a) Protein-chip analysis for culture supernatant of HuMSC-HNF4a and HuMSC-CON. (b) Levels of IL-10 and M-CSF in the culture supernatant of HuMSC-HNF4a and HuMSC-CON. (c) Immunofluorescence of HuMSC-HNF4a and HuMSC-CON in the small intestine (scale bar, $500 \mu \mathrm{m}$ ), liver (scale bar, $100 \mu \mathrm{m}$ ), spleen (scale bar, $50 \mu \mathrm{m}$ ), and lung (scale bar, $50 \mu \mathrm{m}$ ), $\mathrm{n}=3$ mice per group. (d) Serum levels of TNF- $a$ and IL- $1 \beta$ in the mouse ALF model $(n=4-6)$. All data are shown as the mean \pm SD. ${ }^{*} p<0.01$.

\section{$\mathbf{a}$}
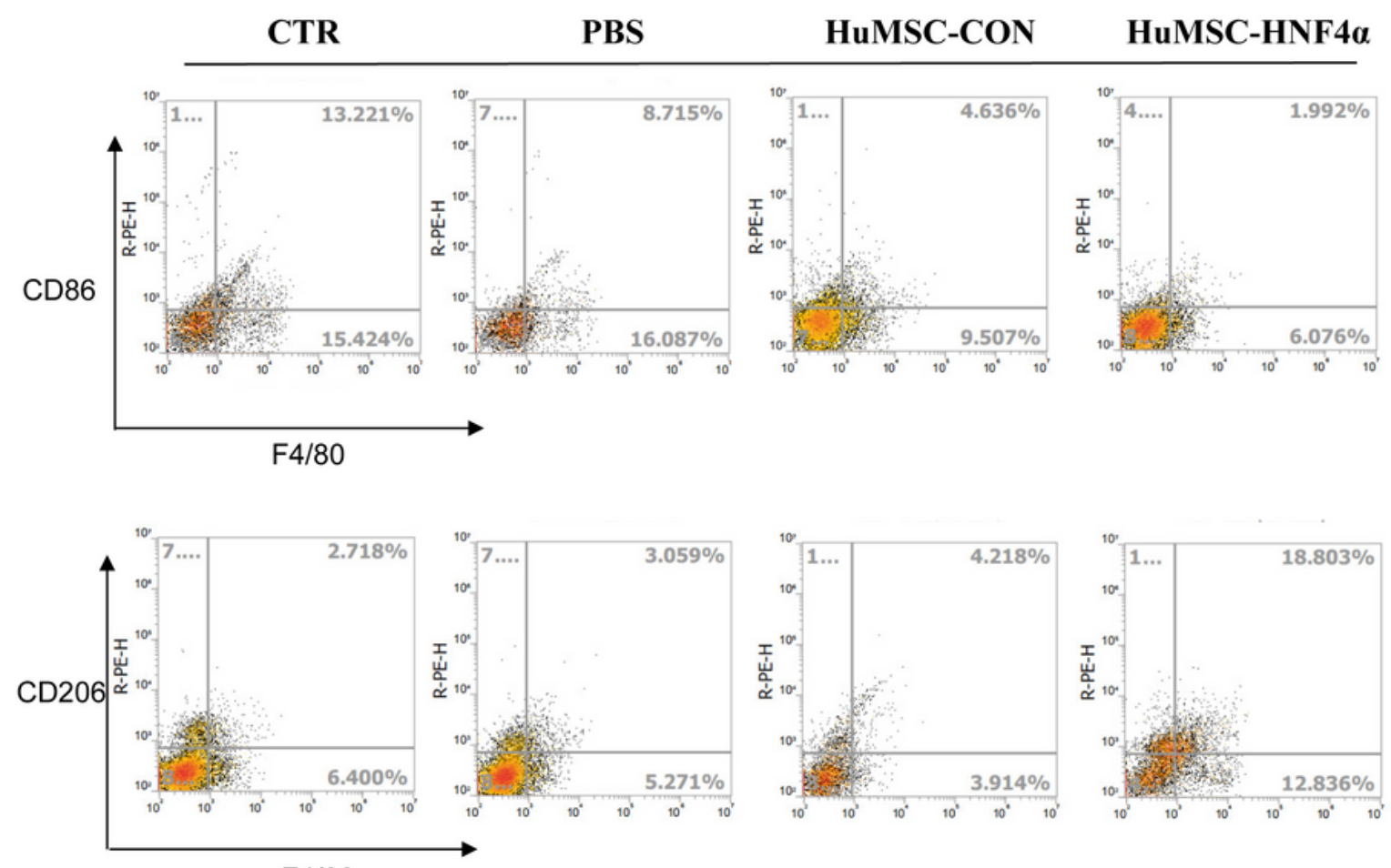

F4/80
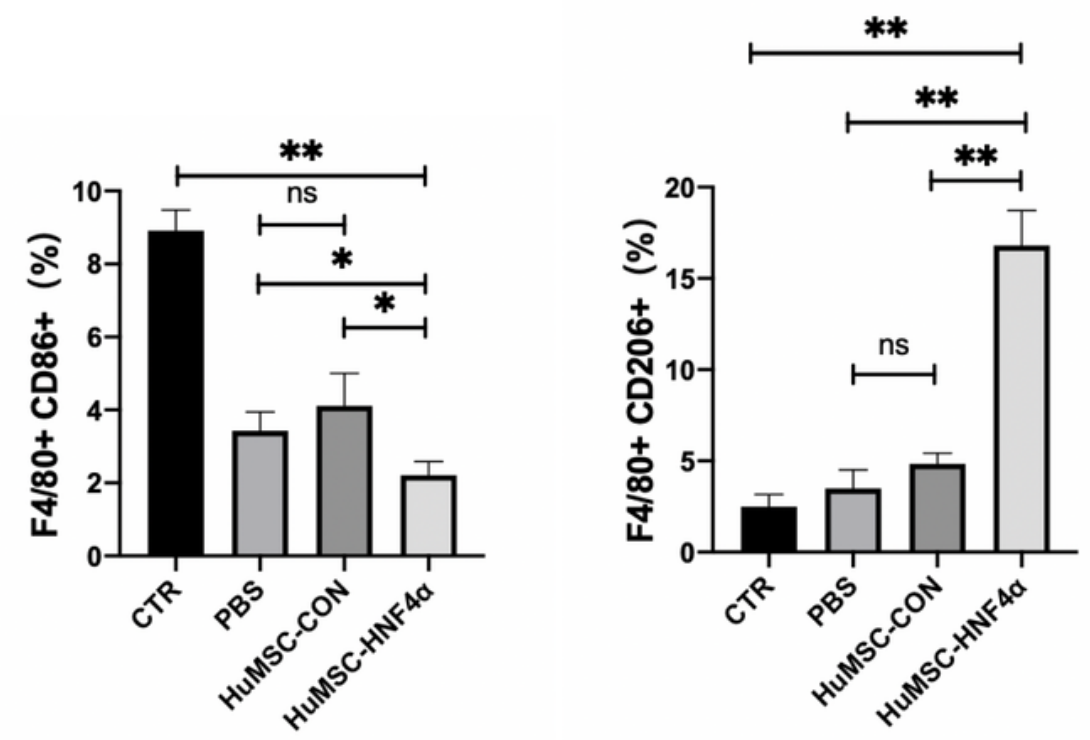

Figure 7 
Overexpression of HNF4a in HuMSCs promoted macrophage polarization toward the M2 type in vivo. (a) Flow cytometry to show the levels of CD86 and CD206 in liver tissues, markers of macrophages, in the mouse model, $n=3$ mice per group. All data are shown as the mean $\pm S D$. $=\star p<0.05, * \star p<0.01$, and ns not significant.

\section{Supplementary Files}

This is a list of supplementary files associated with this preprint. Click to download.

- fig.S1.tif 